\title{
Using a Simple Chair-Side Copy Denture Technique in the AvaDent Digital Denture Process: A Case Report and Review
}

\author{
Amit Paryag, Reisha Rafeek*, Ashley Meighan \\ School of Dentistry, Faculty of Medical Sciences, The University of the West Indies, St. Augustine, Trinidad \& Tobago \\ Email: *reisha.rafeek@sta.uwi.edu
}

How to cite this paper: Paryag, A., Rafeek, R. and Meighan, A. (2018) Using a Simple Chair-Side Copy Denture Technique in the AvaDent Digital Denture Process: A Case Report and Review. Open Journal of Stomatology, 8, 241-249.

https://doi.org/10.4236/ojst.2018.87022

Received: June 1, 2018

Accepted: July 2, 2018

Published: July 5, 2018

Copyright ( 92018 by authors and Scientific Research Publishing Inc. This work is licensed under the Creative Commons Attribution International License (CC BY 4.0).

http://creativecommons.org/licenses/by/4.0/

\begin{abstract}
Introduction: Several techniques have been described by which a pair of dentures may be replicated. Case Report: The method described in this paper used materials readily available in a private practice to generate a pair of duplicate dentures for a 74-year-old Black male, which captured all of the features of the patients' existing pair. These were modified chair-side and used to capture vital information on fit, occlusion and aesthetics, forming a template which was transferred to the laboratory and used to generate new dentures via the traditional laboratory process. The same template was also used to generate a pair of "AvaDent Digital Dentures" by digital scanning and production of a digital denture template for try and then fabrication of the digital denture. Conclusion: The results of this pilot study show that this simple chair-side method of fabricating duplicate dentures can be incorporated successfully into the AvaDent Digital Denture fabrication process to generate a pair of dentures with comparability if not superior fit and aesthetics to a pair fabricated from the same template via the traditional laboratory process.
\end{abstract}

\section{Keywords}

Digital Dentures, Duplicate, Chair-Side, Complete Dentures

\section{Introduction}

Private dental practitioners are often presented with patients requiring replacement of an old pair of complete dentures. Many factors impact upon the ability of the clinician to provide a new pair of dentures which are not only technically and clinically acceptable, but are also acceptable to the patient. Often there are features on the old denture that the clinician wishes to replicate on the new pair 
of dentures. Several techniques have been described by which a pair of dentures may be replicated.

The method described in this paper used materials readily available in a private practice such as heavy-body polyvinyl siloxane (PVS) and polymethyl methacrylate (PMMA) to generate a pair of duplicate dentures which captured all of the features of the patients' existing pair. These were modified chair-side to capture vital information on fit, occlusion and aesthetics, forming a template which was transferred to the laboratory. The information was used to generate new dentures which replicated all of the patient's desired features of the old pair, while incorporating the necessary modifications. The template was also used in the AvaDent digital denture fabrication process to generate a pair of AvaDent Digital Dentures.

\section{Case Report}

A 74-year-old Black male patient presented to the School of Dentistry Polyclinic complaining of dentures that were loose but he was comfortable with the appearance although the teeth were slightly worn down. His medical history was clear and after discussion with the patient it was agreed that the dentures should be copied and the copies modified to meet his requirements. The copy dentures would then be duplicated via traditional and digital denture fabrication processes. The patient's existing dentures (Figures 1(a)-(c)) were taken and copied usingheavy body polyvinyl siloxane (PVS) (VP Mix Putty, Henry Schein, New York, USA).

The patient's dentures were coated in petroleum jelly to act as a separating medium. The heavy body PVS was mixed as per manufacturer's instructions and the fitted surface of the maxillary denture was pressed into the putty until the denture was covered to a position half way down the flange. The edge of the putty was adapted with finger pressure against the denture and the denture held in place until the putty set. (Figure 2(a)). The denture was kept embedded in the putty and petroleum jelly was applied again to all exposed surfaces of denture and putty-mould. A fresh set of heavy body PVS was mixed and applied to the exposed denture surfaces and adapted against the flange and against the putty mould. When this was set it was gently separated and the denture removed giving a mould for the maxillary denture. The process was repeated for the mandibular denture (Figure 2(b), Figure 2(c)). Vents were created in the side of the
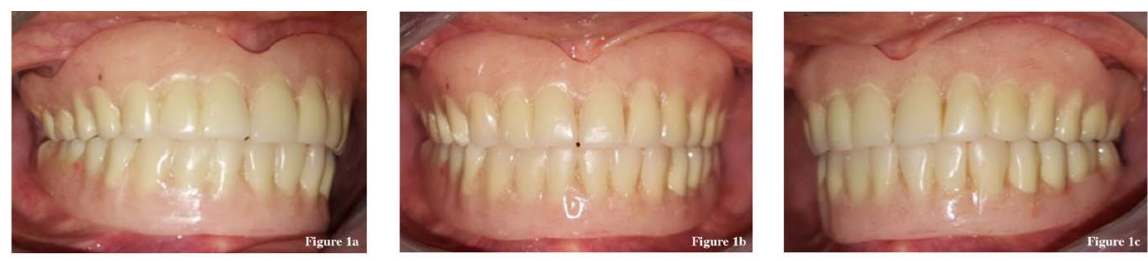

Figure 1. Patient's existing dentures which were used to make copy dentures. (a) Right buccal view (b) Anterior view (c) Left buccal view. 

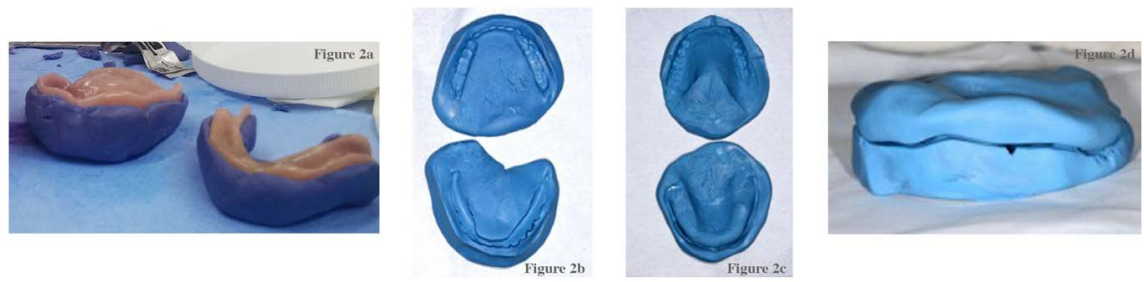

Figure 2. Copying the patient's dentures with putty. (a) Showing patient's dentures pressed down into the putty; (b) Mandibular impressions (c) Maxillary impressions; (d) Shows the "V" shaped vent created.

moulds by cutting "V-Shaped" notches using a scalpel, to allow escape of excess copy material. (Figure 2(d)).

Polymethyl methacrylate (Bosworth Trim, Gibbstown, NJ, USA) was mixed to a smooth runny consistency and poured into one half of each mould (Figure $3(a))$. The other half of the mould was placed on top and pressure applied till the sides of the mould were fully approximated (Figure $3(\mathrm{~b})$ ). The mould was secured with rubber-bands and the PMMA allowed reaching full set. The rubber bands were removed and the PMMA copy denture templates taken from the mould (Figure $3(\mathrm{c})$ ). Sharp edges and the excess material from the vents was trimmed with acrylic burs and the templates polished. The copy denture templates were inserted into the patient's mouth and modified for snugness of fit with Soft reline Material to attain proper retention (Figure 3(d)). PMMA was then added to the occlusal surface of the maxillary copy denture and allowed to reach doughy consistency. The patient was asked to close slowly attain the new vertical dimension he wanted and the position was maintained until the material was fully set.

The resultant copy dentures in polymethyl methacrylate, captured the features of the old dentures as desired by the patient. They were also easily modified for fit and increased vertical dimension of occlusion and the patient allowed functioning in them for one hour on clinic.

The information recorded was sent to the in house laboratory and articulated (Figure 4(a), Figure 4(b)) and a wax trial of the dentures was fabricated, inserted and compared with the old dentures and modified copy dentures, to determine if they adequately met the patient's needs.

These were then processed and delivered to the patient (Figures 5(a)-(c)).

Subsequent to the delivery of the conventionally made dentures, the copy denture in PMMA was sent to the Drake Precision Dental laboratory (Figure 6(a)) (Charlotte, NC, USA) and a digital impression of this was taken (Figure 6(b)).

A digital denture template was returned for trial in the patient before the final denture was made. Upon trying in the digital template, the retention was acceptable, and soft reline was added to ensure snugness of fit (Figures 7(a)-(c)).

The patient used this template for two weeks, and then it was returned to the AvaDent laboratory for fabrication of the final dentures. The final digital denture was delivered, and the patient continues to be reviewed (Figures 8(a)-(c)). 

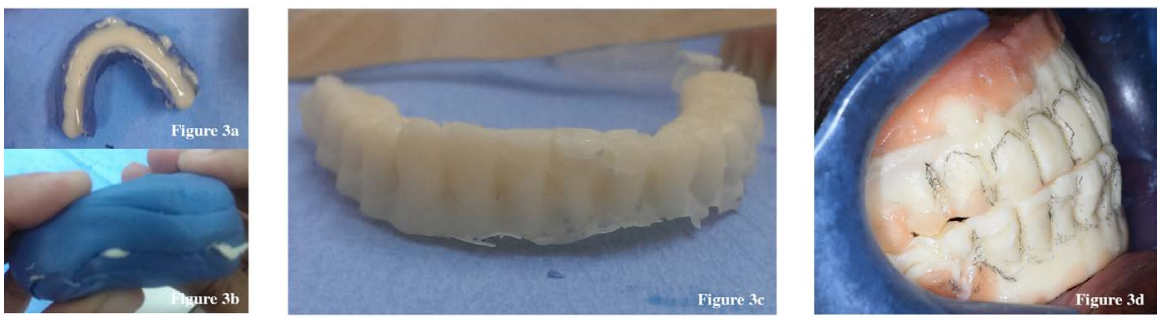

Figure 3. Copy denture templates. (a) Shows the poly-methyl-methacrylate material poured into the mould after removing the patient's denture; (b) Shows the top half of mould applied with pressure and excess material escaping; (c) Shows the resultant copy denture template in poly-methyl-methacrylate before polishing; (d) Shows the copy denture templates inserted into the patients mouth.
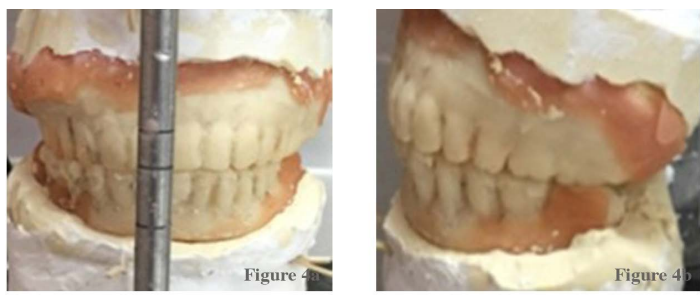

Figure 4. Articulated copy denture templates (a) Anterior view (b) Left buccal view.
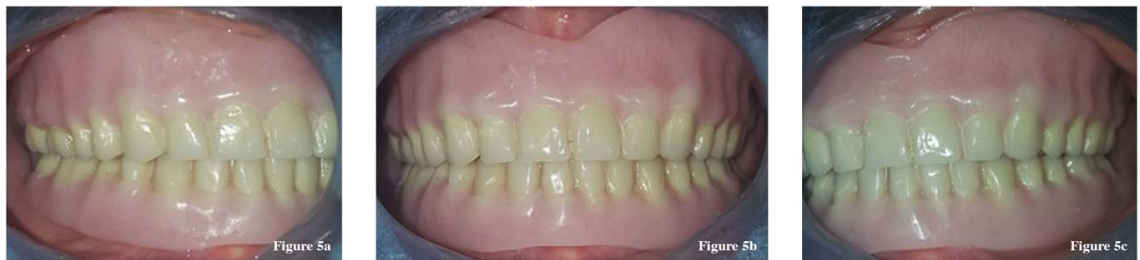

Figure 5. New dentures traditionally fabricated using the copy denture technique fitted (a) Right buccal view (b) Anterior view (c) Left buccal view.
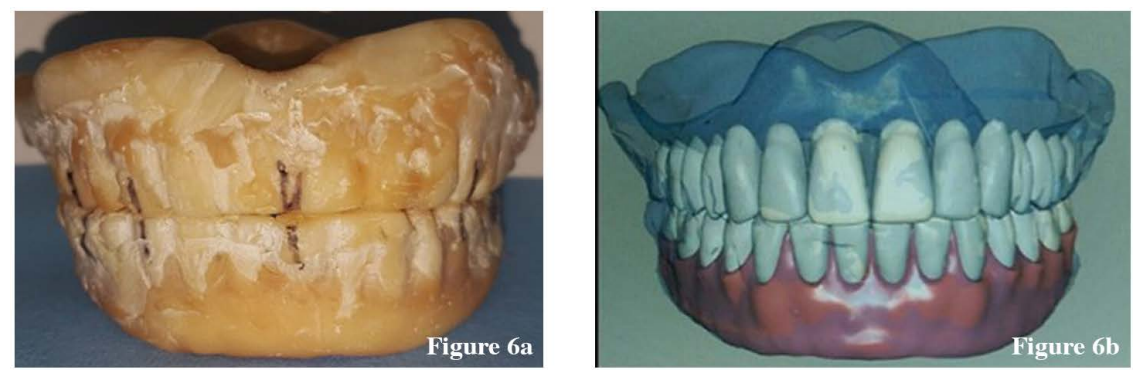

Figure 6. Copy denture. (a) Copy denture which was sent to the AvaDent laboratory; (b) The digital impression taken of it.
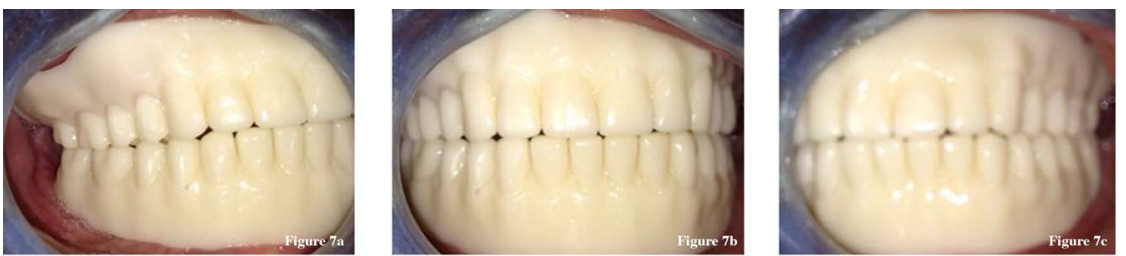

Figure 7. Digital denture template returned from the AvaDent laboratory. (a) Right buccal view; (b) Anterior view; (c) Left buccal view of the template tried in the patients mouth. 

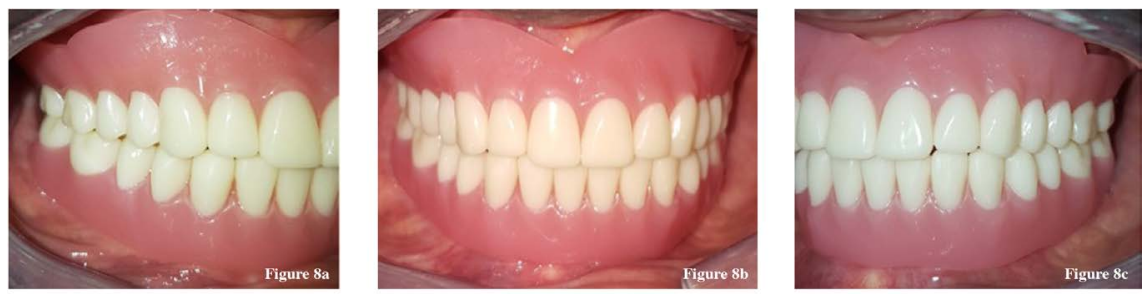

Figure 8. Final digital dentures. (a) Right buccal view; (b) Anterior view; (c) Left buccal view.

\section{Review and Discussion}

The case patient was able to use the traditionally made denture and the AvaDent digital denture, which were both fabricated from the same copy denture. He wore the conventionally made denture first, and was pleased with the outcome as it replicated many of the features he liked from his old denture. The patient was also very pleased with AvaDent digital denture, because of the fit and aesthetics. One of the most common advantages of a digital denture, when compared to a conventionally made denture for the same patient, is that there is a significant increase in retention [1]. The case patient had a similar experience because once the maxillary complete digital denture was in place, it was observed that the retention was superior to that of the conventionally made denture.

The inherent challenges in the fabrication of a new pair of complete dentures are known to dental practitioners. These challenges can prove to be particularly difficult, when the replacement denture is for an elderly patient, or patients with systemic disorders such as Parkinson's, Dementia, Alzheimer's or the physically frail [2]. It may seem that if a patient already possessed a pair of dentures, which they wore for some time, that fabrication of a new pair of dentures would be relatively easy. However, even though previous denture use may enable a patient to adapt more quickly to a new set of dentures, as compared with a patient who has never worn dentures before, this situation can also present unique challenges. When patients are wearing old and inadequate prosthesis over long periods, the necessary muscular control develops and enables them to still function. Therefore, when replacement dentures are made, adaptation is required to relearn this control, and that is where the difficulty arises if major changes are made. For this reason, it is important to identify the patients who may find it difficult to adapt, and copy the relevant features of the old denture to ensure their comfort [2].

In several instances, the expectations of patients regarding aesthetics and fit may be such that they desire the new pair of dentures to look and feel like the old pair, while still meeting their need for change and improvement upon the deficiencies of the old dentures. Often when the new pair of dentures are finished for delivery, the patients express dissatisfaction with the fit and aesthetics, despite having had the opportunity to see the changes made in the new dentures at the teeth in wax or wax trial stage. For this reason, the advantage of a copy denture would be the ease with which neuromuscular adaption to the new dentures can occur. Additionally, as outlined by Vohra and Habib 2013, there would 
be a decrease in the number of visits, a quicker fabrication time, easier jaw relation records, cost effectiveness, and an increase in information transfer to technicians about soft tissue aesthetics, tooth size and position.

There are several methods that can be used to duplicate a denture. In selecting one of the many methods, it is important to consider the intended use of the copy denture, the degree of accuracy required, and the materials and time available to the clinician [3]. The most frequent intended use for a copy denture is as a replacement for an old or worn denture, but it can also be used as a provisional denture during laboratory repair. Other uses as outlined by Wagner 1987 include a provisional denture altered for the release of medication; a provisional denture intended for tissue conditioning; to reline or rebase an existing denture; to serve as an impression tray for a definitive impression; and, as a provisional denture to be tested in the patient to ensure that the vertical dimension, aesthetics, and fit are satisfactory before a final denture is fabricated [3].

Regardless of what the intended use is for the copy denture, the techniques for fabricating one, as summarized by Polyzois et al. 1986 [4], includes making a flexible mold of the original denture using either irreversible hydrocolloid or silicone. When using a silicone such as heavy body polyvinyl siloxane, it is at the discretion of the clinician if it needs to be done with or without a stock tray or copy jig [5]. Once the mold is formed, the replica denture is made by pouring wax, auto polymerizing acrylic resin, or shellac into it. One of the most important factors to consider when assessing a copy denture is the dimensional accuracy of it [4]. In the case of a wax replica, this wax copy can be tried into the patient's mouth once set and the required modifications can be made. Common modifications include adding wax to the occlusal surfaces to correct the occlusal vertical dimension, and also the fitting surface can be relined with light body polyvinyl siloxane to ensure better adaptation and fit [6].

Alternatively, the copy denture can be made with an auto-polymerising acrylic resin as is described by Cooper and Watkinson 1976 [7] and Marcroft et al. 1961 [8]. Polyzois et al. 1986 [4] highlighted that better dimensional accuracy is obtained when the copy denture is made from only modeling wax or an acrylic base with wax teeth when compared to an all acrylic resin replica. However, there are shortcomings associated with making a denture base from modeling wax, as this might distort so it would need to be assessed in the mouth promptly [4].

Bearing these shortcomings in mind, when the patient in the case presented to the School of Dentistry requiring a new set of dentures, an assessment was done to determine if copying the dentures would be feasible. A proper assessment is necessary to determine if any modifications need to be made to the occlusal, polished or fit surfaces of the dentures before the copy denture is made [5]. Having ascertained this, the patient's consent was obtained and a process of copying the dentures as described below was followed mimicking conditions as they may occur within the private practice environment.

Once an acceptable replica is made it is usually used to generate the new den- 
tures via the traditional laboratory process. In this pilot study, the copy denture was also incorporated into the Avadent digital denture process to fabricate the new dentures.

Digital dentures or Computer-aided design/computer-assisted manufacturing (CAD/CAM) dentures are more becoming more popular now when fabricating complete dentures. In 2014, Lozada et al. [9] described the numerous advantages as follows: 1) as little as two appointments required to provide a denture, 2) a reduced amount of residual monomer, 3 ) increased accuracy in the denture fit as it is fabricated from pre-polymerized denture base resin under high pressure, and 4) a collection of digital data that can be used to fabricate an extra denture with the same base morphology and tooth positions in the event that the original one is lost or damaged. New technology applications are often an exciting part of research, but it may also be daunting to some clinicians to have to learn new clinical procedures. Another disadvantage to the digital denture process is that impressions and records have to be mailed to a digital denture manufacturing company, rather than having the usual convenience of a local denture laboratory. Also important to note is that the costs for fabricating digital dentures and conventional dentures is comparable, depending on your location [9].

Schwindling and Stober 2016 [10] and Kattadiyil et al. 2015 [1] described that there was no significant difference in phonetics and aesthetics between digital dentures and other types of complete dentures. Similarly, the case patient described that there was no noticeable difference in phonetics. However, the case patient did express a preference for the aesthetics of the digital denture.

After two weeks of wearing the digital denture, upon review the case patient described that the most noticeable difference was that the digital denture was much lighter, and as a result more comfortable to wear. Patient satisfaction was expected, as Bidra et al. 2016 [11] described an overall patient satisfaction of 79\% (11/14 participants). Kattadiyil et al. 2015 [1] also highlighted that there was an $80 \%$ satisfaction rate (12/15 participants).

Another common advantage to digital dentures is that less clinical time is needed [12]. When the case patient's copy denture was mailed, a template trial digitally milled was sent back prior to the final digital denture. As no adjustments were made to the template trial, one can deduce that this visit for the template trial was not completely necessary. If the template trial step was removed, then that would further reduce the necessary clinical time required when using a copy denture in the AvaDent digital denture process.

The case patient will also be able to have an extra or replacement denture made, when the current denture becomes worn or damaged. That is a benefit of the digital denture process, because a database of all the relevant information is stored to be used at a future time if necessary [12].

Some of the frequently reported shortcomings of digital dentures include issues with occlusal vertical dimensions, problems with aesthetics, and the need for immediate relines [12]. These were not experienced by the case patient, but the favorable outcomes may be due in part to careful patient selection. 


\section{Conclusion}

The results of this case report show that this simple chair-side method of fabricating duplicate dentures can be incorporated successfully into the Digital Denture Fabrication process to generate a pair of dentures with comparability if not superior fit and aesthetics to a pair fabricated from the same template via the traditional laboratory process.

\section{References}

[1] Kattadiyil, M.T., Jekki, R., Goodacre, C.J. and Baba, N.Z. (2015) Comparison of Treatment Outcomes in Digital and Conventional Complete Removable Dental Prosthesis Fabrications in a Predoctoral Setting. Journal of Prosthetic Dentistry, 114, 818-825.https://doi.org/10.1016/j.prosdent.2015.08.001

[2] Vohra, F.A. and Habib, S.R. (2013) Copy Denture, Existing Dentures Replacing Technique Geriatric Patient. Journal of the Pakistan Dental Association, 22, 265-270.

[3] Wagner, A.G. (1987) A Temporary Replacement for an Existing Complete Denture. Journal of Prosthetic Dentistry, 58, 522-525. https://doi.org/10.1016/0022-3913(87)90287-3

[4] Polyzois, G.L., Stavrakis, G.A. and Demetriou, P.P. (1986) Dimensional Accuracy of Duplicate Dentures Prepared by Different Methods. Journal of Prosthetic Dentistry, 55, 513-517. https://doi.org/10.1016/0022-3913(86)90190-3

[5] Soo, S. and Cheng, A.C. (2014) Complete Denture Copy Technique-A Practical Application. Singapore Dental Journal, 35, 65-70.

https://doi.org/10.1016/j.sdj.2013.12.001

[6] Basker, R.M., Davenport, J.C. and Thomason, J.M. (2011) Prosthetic Treatment of the Edentulous Patient. 5th Edition, Wiley-Blackwell, Hoboken, New Jersey.

[7] Cooper, J.S. and Watkinson, A.C. (1976) Duplication of Full Dentures. British Dental Journal, 141, 344-348. https://doi.org/10.1038/sj.bdj.4803844

[8] Marcroft, K.R., Tencate, R.L. and Hurst, W.W. (1961) Use of a Layered Silicone Rubber Mold Technique for Denture Processing. Journal of Prosthetic Dentistry, 11, 657-664. https://doi.org/10.1016/0022-3913(61)90173-1

[9] Lozada, J.L., Garbacea, A., Goodacre, C.J. and Kattadiyil, M.T. (2014) Use of a Digitally Planned and Fabricated Mandibular Complete Denture for Easy Conversion to an Immediately Loaded Provisional Fixed Complete Denture. Part 1. Planning and Surgical Phase. International Journal of Prosthodontics, 27, 417-421.

https://doi.org/10.11607/ijp.3825

[10] Schwindling, F.S. and Stober, T. (2016) A Comparison of Two Digital Techniques for the Fabrication of Complete Removable Dental Prostheses: A Pilot Clinical Study. Journal of Prosthetic Dentistry, 116, 756-763.

https://doi.org/10.1016/j.prosdent.2016.03.022

[11] Bidra, A.S., Farrell, K., Burnham, D., Dhingra, A., Taylor, T.D. and Kuo, C. (2016) Prospective Cohort Pilot Study of 2-Visit CAD/CAM Monolithic Complete Dentures and Implant-Retained Overdentures: Clinical and Patient-Centered Outcomes. Journal of Prosthetic Dentistry, 115, 578-586. https://doi.org/10.1016/j.prosdent.2015.10.023

[12] Kattadiyil, M.T. and AlHelal, A. (2017) An Update on Computer-Engineered Com- 
plete Dentures: Literature Review on Clinical Outcomes. Journal of Prosthetic Dentistry, 117, 478-485

https://doi.org/10.1016/j.prosdent.2016.08.017

https://doi.org/10.1016/j.prosdent.2016.12.006 